\title{
Screening and managing a low-risk pregnant population using continuous-wave Doppler ultrasound in a low- income population: A cohort analytical study
}

\author{
S Nkosi, FCOG (SA), MMed; J Makin, MB BCh, BSc (Epidemiology), MSc (Clinical Epidemiology); T Hlongwane, FCOG (SA), \\ MMed (Obstetrics and Gynaecology); R C Pattinson, FRCOG, MD, FCOG (SA), MMed (Obstetrics and Gynaecology), MB BCh, BSc
}

South African Medical Research Council Maternal and Infant Health Care Strategies Unit, Faculty of Health Sciences, University of Pretoria, South Africa

Corresponding author: S Nkosi (spencernkosi@gmail.com)

\begin{abstract}
Background. In South Africa (SA), the largest category of perinatal deaths is unexplained stillbirths. Two-thirds of these occur in the antenatal period and most fetuses are macerated, but at antenatal clinics the mothers were generally regarded as healthy, with low-risk pregnancies. Innovative methods are urgently required to detect fetuses at risk of stillbirth and manage the mothers appropriately.

Objectives. To determine the prevalence of raised resistance indices (RIs) of the umbilical artery in a low-risk, low-income population and ascertain whether use of this information can prevent perinatal deaths.

Methods. A descriptive study was performed in Mamelodi township, east of Pretoria, SA, on pregnant women attending antenatal clinics draining to two community health centres (CHCs). These women, classified as having low-risk pregnancies, were screened for placental insufficiency using a continuous-wave Doppler ultrasound apparatus (Umbiflow) between 28 and 32 weeks' gestation. When a raised RI was detected, the mother was referred to a high-risk clinic and managed according to a standard protocol. A cohort analytical study compared women who attended antenatal care at the same clinics as the Umbiflow group but did not have an Umbiflow test with those who had an Umbiflow test. The outcomes of all the deliveries in Mamelodi were recorded. The prevalences of abnormal RIs, absent end-diastolic flow (AEDF), stillbirths and neonatal deaths were the main outcome measures.

Results. An Umbiflow RI was performed in 2868 women, and pregnancy outcome was available for 2539 fetuses (88.5\%); 297 fetuses $(11.7 \%)$ were regarded as at high risk. AEDF was found in $1.5 \%$ of the population screened with an outcome. There were 29 perinatal deaths in the Umbiflow group (low risk $n=18$, high risk $n=11$ ). The perinatal mortality rate for 12168 women attending the CHCs and the antenatal clinics draining to the CHCs who did not have an RI was 21.3/1 000 births, significantly higher than that in the Umbiflow group (11.4/1 000 births) (risk ratio $0.58,95 \%$ confidence interval $0.42-0.81$ ).

Conclusions. The prevalence of AEDF in this low-risk population is $\sim 10$ times higher than that previously recorded. Use of the information prevented a number of perinatal deaths, most of which would have been macerated stillbirths. Screening a low-risk pregnant population using continuous-wave Doppler ultrasound may substantially reduce the prevalence of unexplained stillbirths in SA.
\end{abstract}

S Afr Med J 2019;109(5):347-352. DOI:10.7196/SAMJ.2019.v109i5.13611

It is estimated that a substantial proportion $(30-50 \%)$ of fetal deaths are attributable to fetal growth restriction (FGR). ${ }^{[1]}$ Approximately $25 \%$ of children born in low- and-middle-income countries (LMICs) are small for gestational age (SGA). ${ }^{[2]}$ Babies who are SGA are at increased risk of mortality and neonatal morbidity. ${ }^{[3,4]}$ The SGA fetus may be small but healthy, or the condition may be due to pathological growth failure, i.e. FGR. ${ }^{[5]}$ Some babies classified as appropriate for gestational age (AGA) may also be growth restricted, and a test to detect placental insufficiency would detect these fetuses as well ${ }^{[1]}$ and be preferable to measuring size alone. In South Africa (SA), the largest category of perinatal deaths is unexplained stillbirths. A quarter of these fetuses are SGA, two-thirds of the deaths occur in the antenatal period, and most of the mothers are healthy and classified as having low-risk pregnancies. ${ }^{[6]}$

The use of Doppler ultrasound for assessment of blood flow in fetal umbilical vessels can quantify placental function and be used to identify placental insufficiency in both SGA and AGA fetuses. Abnormal flow indices correlate with intrauterine growth retardation and adverse fetal and neonatal outcomes, particularly when absent or reversed end-diastolic flow is identified. Cochrane reviews on the use of Doppler ultrasound in high-risk pregnancies ${ }^{[7]}$ indicate that it can reduce perinatal mortality and optimise the application of obstetric interventions. However, there have been only five trials in low-risk or unselected pregnancies. ${ }^{[8]}$ There is insufficient evidence to establish whether use of Doppler ultrasound has benefits in these populations, and no trials have been conducted in unselected populations of women in LMICs.

\section{Objectives}

To ascertain the prevalence of raised resistance indices (RIs) of the umbilical artery in a low-risk, low-income population and whether use of this information can prevent perinatal deaths.

\section{Methods}

A descriptive study investigated the prevalence of abnormal RIs and absent end-diastolic flow (AEDF) of the umbilical artery detected by the Umbiflow apparatus (Council for Scientific and Industrial Research (CSIR), SA), a hand-held, mobile continuouswave Doppler ultrasound device, in a low-risk and low-income pregnant population, and a cohort analytical study examined the 
effect of using the information in managing the pregnancies.

Mamelodi township, east of Pretoria, SA, has a population of $\sim 410000$ people with 54000 households (half living in rooms or shacks). About a third of the population is unemployed and $20 \%$ of households are food insecure. There are three healthcare delivery sites, Mamelodi Hospital, where the majority of births take place, and two community health centres (CHCs), Stanza Bopape and Dark City, that also conduct births and refer to Mamelodi Hospital. There are $\sim 10000$ births per year in all the facilities. Mamelodi Hospital has two obstetricians, one of whom (SN) saw all the women referred with abnormal Umbiflow tests. The hospital can do caesarean deliveries at all times. It refers tertiary cases to Steve Biko Academic Hospital. The CHCs are run by midwives, as are the antenatal clinics draining to them. Women with high risk factors are referred to the Mamelodi Hospital high-risk antenatal clinic for further follow-up. Pregnant women are classified as having a low-risk or high-risk pregnancy according to the criteria of focused antenatal care described by the World Health Organization (WHO). ${ }^{[9,10]}$ They have five antenatal visits: booking and at 20, $26-28,32-34$ and 38 weeks' gestation. They are referred to the hospital if they have not delivered by 41 weeks. HIV-positive women are only classified as being at high risk if there is a complication associated with the HIV infection, e.g. tuberculosis.

Women attending the CHCs between 28 and 32 weeks' gestation or with a symphysisfundal (SF) measurement of $26-30 \mathrm{~cm}$ if the gestational age was unknown were asked to participate in the study and screened for placental insufficiency using the Umbiflow. In all cases there was a printout of the waveforms so that the quality of the Doppler examination could be assessed. The accuracy of the Umbiflow apparatus was previously tested against a commercial unit and the RI readings were very similar. ${ }^{[11]}$

In the cohort analytical study, the outcome of the population screened with Umbiflow testing was compared with that in the population that was not screened but attended antenatal care in the same areas and delivered a singleton baby at $\geq 28$ weeks or a baby weighing $\geq 1000 \mathrm{~g}$ if the gestational age was unknown, and received antenatal care at either of the CHCs or at the antenatal clinics draining to the CHCs.

The Umbiflow RI was classified as either low risk or high risk depending on the value in relation to the RI graph, ${ }^{[12]}$ using a cut-off of the 75th centile. Women with a low-risk result were considered normal and continued with their routine antenatal care. Those with a value $>75$ th centile were considered at high risk and referred to the high-risk clinic at Mamelodi Hospital. To ascertain the false negatives, an author (TH) assessed 226 consecutive pregnant women from a $\mathrm{CHC}$ who had a low-risk Umbiflow RI with conventional ultrasound including pulsed Doppler.

Fig. 1 illustrates the protocol for managing women with an abnormal Umbiflow result. At the high-risk clinic, a specialist (SN) performed a detailed ultrasound examination using a Phillips ultrasound with a curvilinear abdominal probe and pulsed Doppler. A biophysical profile was performed and patients were managed based on a set protocol (Fig. 1). Women with an abnormal RI but with end-diastolic flow were seen weekly for repeat Doppler examinations and fortnightly for growth scans. Women whose fetus had AEDF were admitted; if the fetus was $<34$ weeks' gestation corticosteroids were given and the woman and her fetus were monitored in hospital. Delivery was performed when the pregnancy reached 34 weeks' gestation, if the cardiotocograph became abnormal, or if the mother's condition deteriorated.
Outcome data were obtained from the electronic birth register at the various delivery sites. Umbiflow measurement was performed between 28 and 32 weeks' gestation, so most fetuses would have weighed $>1000 \mathrm{~g}$ at entry to the study. In the Umbiflow group, the expected date of delivery was recorded, and a search was conducted for the woman in the birth register from that date. The search included looking for delivery prior to the expected date to identify women who had delivered prematurely. If the woman was not found in the register, she was phoned and the outcome was recorded; if she could not be contacted by telephone after three attempts at different times of the day and on different days, a home visit was made by the wardbased outreach teams working with the Department of Family Medicine. Only after this was the outcome for the woman regarded as missing.

SGA was defined as <10th centile according to the WHO Fetal Growth Charts. ${ }^{[13]}$ Severe pre-eclampsia was defined as a blood pressure of $160 / 110 \mathrm{mmHg}$ on two occasions 30 minutes apart and $3+$ proteinuria according to urine dipsticks or symptoms of headache, epigastric pain and blurred vision.

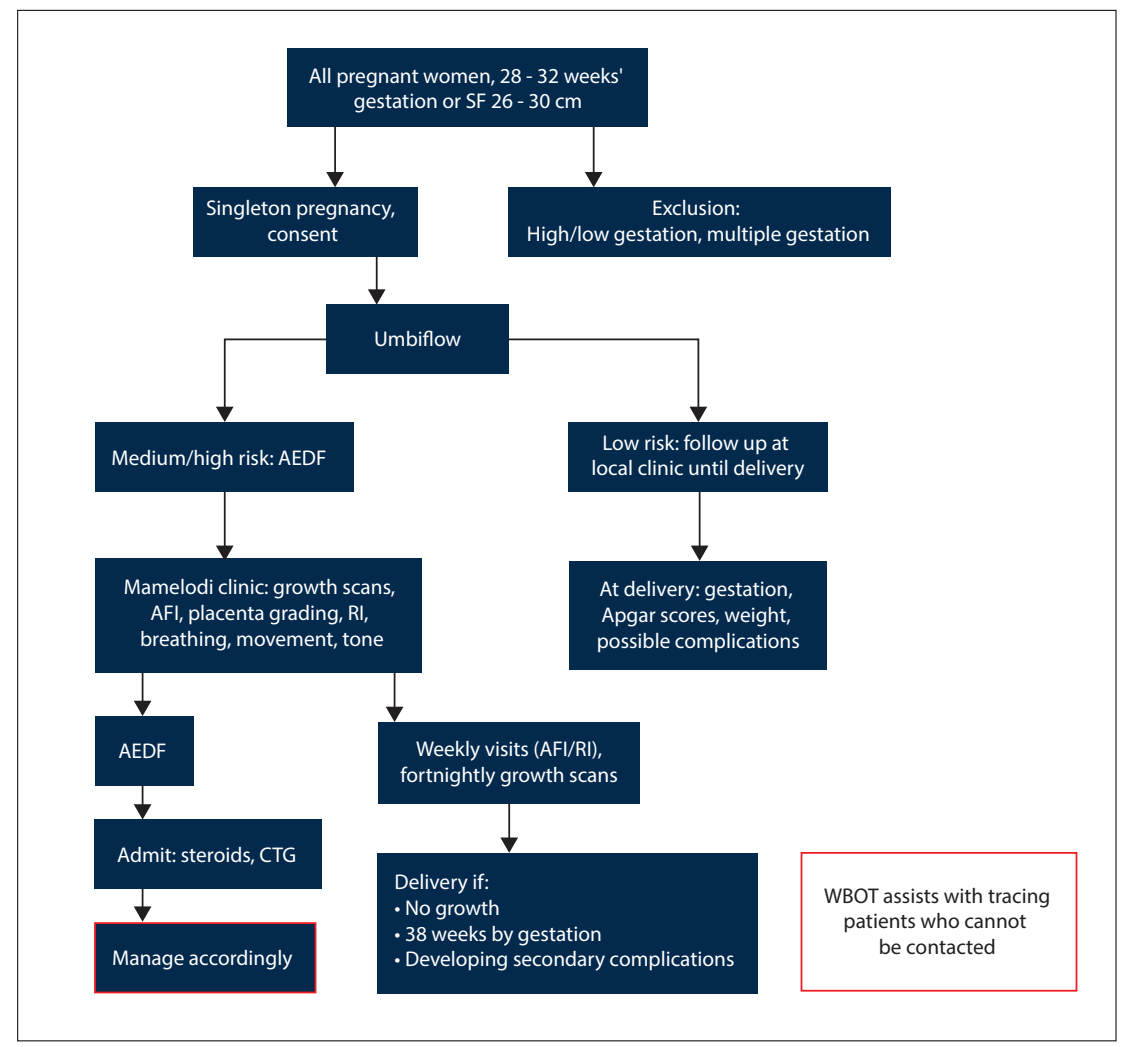

Fig. 1. Protocol for managing women with an abnormal Umbiflow result. $(S F=$ symphysis-fundal height; $A E D F=$ absent end-diastolic flow; $A F I=$ amniotic fluid index; $R I=$ resistance index; $C T G=$ cardiotocograph; WBOT = ward-based outreach team.) 
The project started recruiting women for Umbiflow testing on 1 June 2015 and data collection of births started then and stopped for all births on 31 July 2017. The last Umbiflow was performed on 30 April, allowing time for the women to give birth. Umbiflow tests were done at the two CHCs. Funding for 2 years was allocated for the study and no sample size calculation was performed.

Ethics approval was obtained from the University of Pretoria Faculty of Health Sciences Ethics Committee (ref. no. 473/ 2014). Written consent was obtained from every woman prior to performing the Umbiflow test. The South African Medical Research Council, which was one of the funders, approved the study protocol.

\section{Results}

During the 26-month study period, there were 25918 births at the healthcare delivery sites in Mamelodi. After excluding women at $<28$ weeks' gestation or who gave birth to a baby weighing $<1000 \mathrm{~g}$ if the gestational age was unknown, and those who did not attend antenatal care, were $<18$ years of age or did not attend the clinics at or draining to the CHCs, 15036 pregnancies were analysed (Fig. 2). There were 2242 pregnancies classified as having a low-risk RI (88.3\%) and 297 as having a high-risk RI (11.7\%) with outcomes. Thirty-eight fetuses $(11.8 \%)$ in the high-risk group had or developed AEDF (1.5\% of the population screened with an outcome).

A study within the study was conducted to determine the false-negative rate. A consecutive sample of 226 pregnant women in the Umbiflow group who had a lowrisk Umbiflow RI were also assessed with conventional ultrasound and pulsed Doppler; only 3 had a high-risk result, giving a falsenegative rate of $1.3 \%$, and the specificity was $98.7 \%$ in this sub-set. In the study group as a whole, 32 women had a high-risk Umbiflow RI but a low-risk RI on the pulsed Doppler, giving a false-positive rate of $9.0 \%$ (32/355 Umbiflow high-risk cases). These 32 patients were referred back to the clinic for routine antenatal care. The sensitivity was $91.0 \%$ for the whole study.

Table 1 sets out the demographics of the Umbiflow population. The high-risk group had a slightly older age distribution than the low-risk group. As expected, the high-risk group had more preterm babies and more SGA babies, but unexpectedly there were fewer HIV-positive women than in the lowrisk group and less severe pre-eclampsia.

The Umbiflow group was similar to the cohort analytical study control group

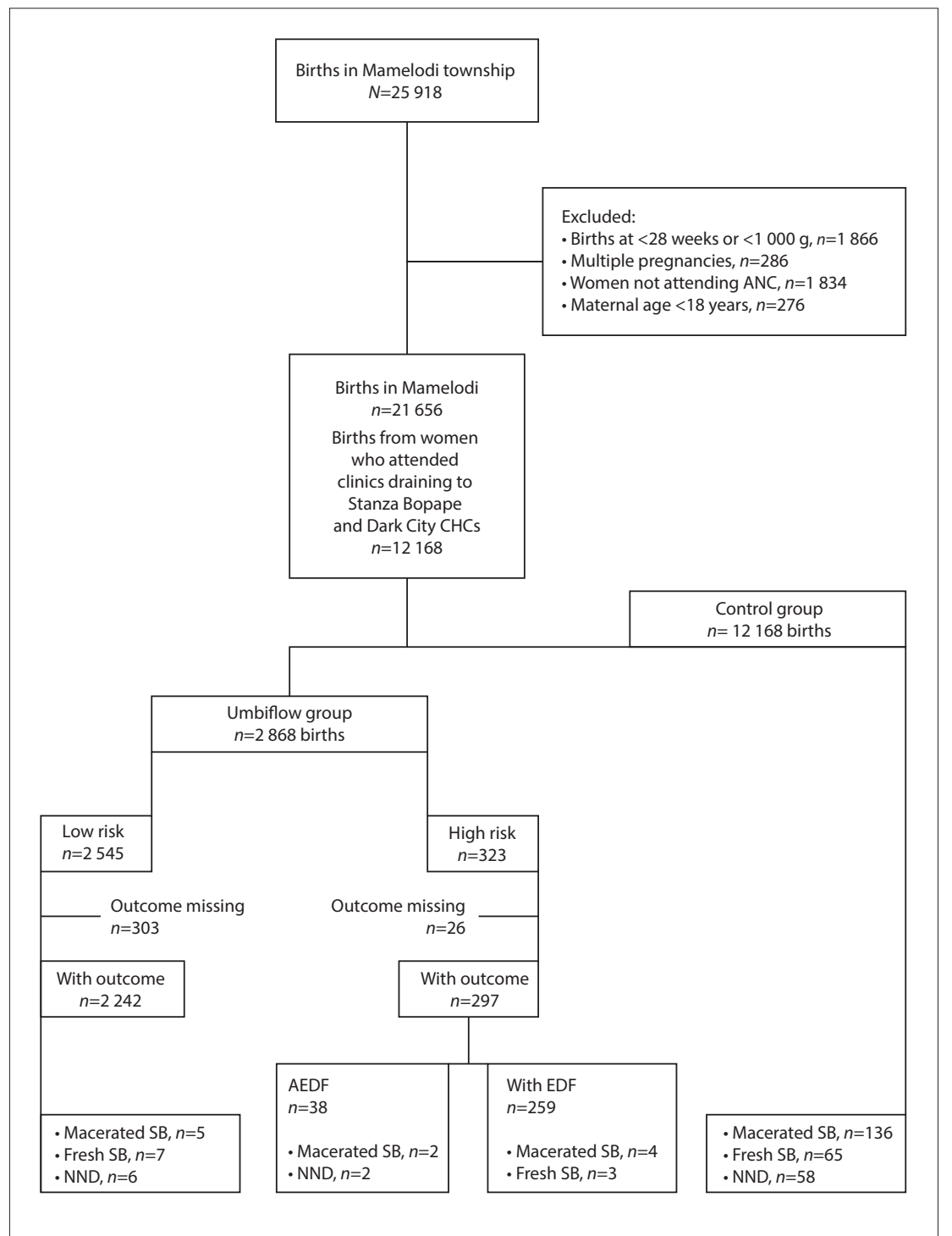

Fig. 2. Flow-chart of cases included in the study. $(A N C=$ antenatal care; $S B=$ stillbirth, $N N D=$ neonatal death, $A E D F=$ absent end-diastolic flow, $E D F=$ end-diastolic flow. $)$

in maternal age, parity, gestational age at delivery, low birth weight and HIV status, but had significantly fewer SGA babies and less severe pre-eclampsia (Table 2).

The outcomes in the Umbiflow and control groups and the impact of active management are given in Table 3.

There were more inductions overall in the cohort analytical control group than in the Umbiflow group, but as expected significantly more inductions in the highrisk Umbiflow group compared with the low-risk Umbiflow group in the descriptive study. For caesarean deliveries there was no overall difference between the cohort analytical groups, but a very significant difference between the low-risk and highrisk Umbiflow groups.

Overall the perinatal mortality rate was significantly lower in the Umbiflow group compared with the control group, in which most deaths were macerated stillbirths. There were 11 perinatal deaths in the high-risk Umbiflow group. Four fetuses had AEDF: 2 were stillborn after the mother was admitted, but the mothers then discharged themselves and were lost to follow-up, and 2 died in the neonatal period (one was born at 34 weeks and died of sepsis on the 8th day, and the mother of the other discharged herself and was lost to follow-up until she developed severe pre-eclampsia and delivered at 36 weeks; the baby died of sepsis). Seven other high-risk babies with end-diastolic flow died (one due to abruptio placentae and 3 due to congenital abnormalities; the 3 remaining women with stillbirths did not arrive at the high-risk clinic and were lost to follow-up). Ultimately 7 of the 11 women with perinatal deaths in the high-risk groups declined 
Table 1. Demographic information on Umbiflow populations

\begin{tabular}{|c|c|c|c|c|}
\hline & \multicolumn{3}{|c|}{ Umbiflow } & \multirow[b]{2}{*}{$p$-value } \\
\hline & $\begin{array}{l}\text { Low risk }(N=2242 \text {, } \\
88.3 \%), n(\%)\end{array}$ & $\begin{array}{l}\text { High risk }(N=297, \\
11.7 \%), n(\%)\end{array}$ & $\begin{array}{l}\text { Total }(N=2539), \\
n(\%)\end{array}$ & \\
\hline Age (years) & & & & 0.006 \\
\hline$<20$ & $88(3.9)$ & $6(2.0)$ & $94(3.7)$ & \\
\hline $20-34$ & $1757(78.7)$ & $227(76.9)$ & $1984(78.5)$ & \\
\hline$\geq 35$ & $387(17.3)$ & $62(21.0)$ & $449(17.8)$ & \\
\hline Missing data & 10 & 2 & 12 & \\
\hline Parity & & & & 0.044 \\
\hline 0 & $712(31.8)$ & $92(31.0)$ & $804(31.4)$ & \\
\hline $1-4$ & $1471(66.9)$ & $191(67.3)$ & $1662(67.0)$ & \\
\hline$\geq 5$ & $15(0.7)$ & $1(0.4)$ & $16(0.6)$ & \\
\hline Missing data & 44 & 13 & 57 & \\
\hline Gestational age (weeks) & & & & $<0.0001$ \\
\hline $28-33$ & $19(0.9)$ & $16(6.5)$ & $35(1.5)$ & \\
\hline $34-37$ & $501(24.4)$ & $76(30.8)$ & $577(25.1)$ & \\
\hline$\geq 38$ & $1530(74.6)$ & $155(62.8)$ & $1685(73.4)$ & \\
\hline Missing data & 198 & 50 & 248 & \\
\hline LBW $(<2500 \mathrm{~g})$ & $181(8.1)$ & $82(27.6)$ & $263(10.4)$ & $<0.0001$ \\
\hline SGA & $362 / 2036(17.8)$ & $83 / 244(34.0)$ & $445 / 2280(19.5)$ & 0.022 \\
\hline HIV-positive & $530(24.7)$ & $51(18.5)$ & $581(24.0)$ & $<0.0001$ \\
\hline Missing/unknown HIV status & 16 & 3 & 19 & \\
\hline Severe pre-eclampsia/eclampsia & $8(0.36)$ & $2(0.67)$ & $13(0.5)$ & NS \\
\hline
\end{tabular}

Table 2. Comparison between the Umbiflow group and the control group

\begin{tabular}{|c|c|c|c|}
\hline & Umbiflow $(N=2539)$ & Control group $(N=12168)$ & $p$-value \\
\hline Age (years), mean (SD) & $28.6(5.8)$ & $27.7(6.0)$ & NS \\
\hline Parity, mean (SD) & $1.2(1.1)$ & $1.3(1.2)$ & NS \\
\hline Gestational age (weeks), mean (SD) & $38.5(1.7)$ & $38.8(2.0)$ & NS \\
\hline Birth weight $(\mathrm{g})$, mean $(\mathrm{SD})$ & $3068(521)$ & $3049(540)$ & NS \\
\hline LBW (<2 $500 \mathrm{~g}), n(\%)$ & $263(10.4)$ & $1464(12.0)$ & NS \\
\hline SGA, $n(\%)$ & $445 / 2280(19.5)$ & $2905 / 10886(26.7)$ & $<0.0001$ \\
\hline HIV-positive, $n(\%)$ & $581(24.0)$ & $2973(24.4)$ & NS \\
\hline Severe pre-eclampsia/eclampsia, $n(\%)$ & $14(0.5)$ & $136(1.1)$ & $<0.001$ \\
\hline
\end{tabular}

further investigation or treatment and were lost to follow-up. The outcomes of all 7 were traced in the birth register or by telephonic contact.

\section{Discussion}

\section{Main findings}

Routine performance of the Umbiflow RI at 28 - 32 weeks' gestation in a low-risk population identified raised Doppler RIs in $11.7 \%$ of pregnant women. AEDF was found in $1.5 \%$ of the Umbiflow group. This is a $5-10$ times higher prevalence of AEDF than recorded in other studies screening low-risk or unselected populations. ${ }^{[8]}$ To our knowledge, the present study is the first large study using continuouswave Doppler ultrasound to screen a pregnant population classified as at low risk in a low-income setting. Active management of these newly identified high-risk pregnancies improved perinatal outcome significantly compared with the control group in which the Umbiflow test was not performed.

The Umbiflow has been validated against commercial ultrasound machines for the detection of umbilical vessel flow abnormalities. ${ }^{[1]}$ The device does not need a trained and experienced sonographer and can be operated by trained midwives and nurses. Studies have shown that the Umbiflow system can measure the RI of the umbilical blood flow accurately when compared with other devices. ${ }^{[1]}$

\section{Study strengths and limitations}

A cut-off of the 75th centile was used for classifying women as at high risk. ${ }^{[14]}$ Theoretically $25 \%$ of women should have been referred, but only $11.3 \%$ were. The normal RI curves for SA were developed in the 1980s and included the whole population of pregnant women. ${ }^{[12]}$ The curves may not be applicable to the current population, as HIV infection was rare then and the prevalence of cigarette smoking in the population in which the curves were developed was extremely high; ${ }^{[15]}$ alternatively, the low proportion of high-risk Umbiflow RIs may reflect that the group of women screened in this study were truly low risk. Importantly, the women identified as having highrisk RIs were regarded as having uncomplicated pregnancies at the time of the RI and were not identified as having a potential fetal problem. Unexplained stillbirth is the most common category listed for perinatal death in SA, and approximately two-thirds of these fetuses are registered as being macerated, i.e. death is likely to have 
Table 3. Outcome and impact: Comparison between the Umbiflow group and the control group

\begin{tabular}{|c|c|c|c|c|c|}
\hline \multirow[b]{2}{*}{ Complications } & \multicolumn{3}{|c|}{ Umbiflow } & \multirow[b]{2}{*}{ Control $(N=12168)$} & \multirow[b]{2}{*}{$p$-value } \\
\hline & Low risk $(N=2242)$ & High risk $(N=297)$ & Total $(N=2539)$ & & \\
\hline Induction, $n(\%)^{*}$ & $15(0.7)$ & $11(3.7)$ & $26(1.0)$ & $238(2.0)$ & 0.003 \\
\hline Caesarean delivery, $n(\%)^{* *}$ & $372(20.2)$ & $142(50.9)$ & $514(24.3)$ & $3048(25.0)$ & 0.61 \\
\hline Mortality $^{\dagger}$ & & & & & RR $(95 \% \mathrm{CI})$ \\
\hline Total SBs, $n$ (SBR/1 000 births) & $12(5.3)$ & $9(30.3)$ & $21(8.3)$ & $201(16.5)$ & $0.55(0.37-0.80)$ \\
\hline Macerated SBs, $n$ (MSBR/1 000 births) & $5(2.2)$ & $6(20.2)$ & $11(4.3)$ & $136(11.2)$ & $0.40(0.23-0.68)$ \\
\hline Fresh SBs, $n$ (FSBR/1 000 births) & $7(3.1)$ & $3(10.1)$ & $10(4.0)$ & $65(5.3)$ & $0.84(0.49-1.44)$ \\
\hline NNDs, $n$ (NNDR/1 000 live births) & $6(2.7)$ & $2(6.9)$ & $8(3.2)$ & $58(4.8)$ & $0.72(0.38-1.32)$ \\
\hline Perinatal deaths, (PNMR/1 000 births) & $18(8.0)$ & $11(37.0)$ & $29(11.4)$ & $259(21.3)$ & $0.58(0.42-0.81)$ \\
\hline
\end{tabular}

occurred antenatally. ${ }^{[6]}$ A significant proportion of these stillbirths were SGA. ${ }^{[16]}$ The biggest impact of the Umbiflow screening was in preventing macerated/antenatal stillbirths. This finding was to be expected, as raised RIs are associated with placental insufficiency and growth restriction. ${ }^{[5]}$

Screening with the Umbiflow was performed between 28 and 32 weeks' gestation, as the pregnancy could be managed actively if an abnormality was detected, with an expectation of a good chance of neonatal survival with the current facilities at Mamelodi Hospital. Further, the number of SGA stillbirths is highest in the gestational age group 32 - 37 weeks. ${ }^{[16]}$ The peak of stillbirths also occurs between 32 and 37 weeks' gestation, indicating that the potential for preventing stillbirths is considerable.

Once detected, the women with a high-risk RI were managed actively, and mortality rates for the whole Umbiflow group were significantly lower than for the comparison group of women who did not have an Umbiflow RI. This decrease in mortality was not due to an increased intervention rate, as the induction and caesarean delivery rates were similar. However, the cohort analytical control group fell into a higher risk category than the Umbiflow group, as evidenced by the increased prevalence of SGA babies and pre-eclampsia. The increase in pre-eclampsia and SGA babies in the control group may explain the higher mortality rates. A randomised trial would need to be performed to create a true control group.

Fetuses with AEDF not managed actively have a high mortality. ${ }^{[17,18]}$ In this study the fetuses with AEDF were actively managed according to protocol, provided the mother agreed. Most of these fetuses survived, but there were two stillbirths in women who declined further treatment and were lost to follow-up, two neonatal deaths due to a nosocomial infection, and one neonatal death in a woman who declined treatment until she was admitted with severe pre-eclampsia. These unfortunate cases form a natural experiment and indicate the severe prognosis of AEDF.

Despite extensive efforts being made to trace the women, $11.5 \%$ of pregnancies in the Umbiflow group were without outcome data $(8.0 \%$ in the high-risk group, among which there was one case of AEDF). However, this figure is acceptable given that the study was done in a low-income setting with a very mobile population.

\section{Interpretation}

Any implementation of an Umbiflow screening programme will have to ensure that there is an effective system of communication and follow-up as well as the necessary high-care resources. If the seven women described above had agreed to the management protocol, the deaths of their babies might have been prevented. The high-risk
Umbiflow clinic averaged about 10 - 15 cases per week, and this put pressure on the resources available at Mamelodi Hospital. The neonatal unit was also put under increased pressure with an increased number of small babies being delivered. However, despite the high caesarean delivery rate in the high-risk Umbiflow group, there was no overall increase in caesarean deliveries when comparing the caesarean delivery rate between all the women who had an Umbiflow test and those women who did not have the test (control group), so in the context of this study Umbiflow testing did not put extra pressure on these resources.

Use of the Umbiflow was simple for the research nurses to learn, only 2 weeks being needed to master the technique, and with a printout of the result the quality of the recordings could be checked. The false-negative rate was $1.3 \%$ and the false-positive rate $9.0 \%$ compared with pulsed Doppler, indicating that the Umbiflow RI is reliable. It can easily be used to screen a large population and requires minimal resources; however, significant resources would be required for women with an abnormal Umbiflow RI.

Several new questions arise as a result of this study. Is the prevalence of AEDF of the umbilical artery the same in other lowincome settings? Why was the prevalence of AEDF so much higher than in high-income settings? Can a more effective cut-off for referral be determined? What will be the most effective methods to scale up this screening, and at what cost?

The WHO states in its new antenatal care guidelines: ${ }^{[1]}$ 'Accurate low-cost methods for detecting abnormal growth are desirable because ultrasound, the most accurate screening tool, is resourceintensive and not widely available in LMICs.' At present the only way to determine the fetal growth rate at primary care level in SA is by measuring the SF height. A recent study of two-stage routine ultrasound scanning in LMICs found no effect on stillbirths or neonatal mortality or an increase in antenatal attendance, ${ }^{[20]}$ seriously calling into question the role of routine ultrasound in preventing perinatal deaths. Screening using continuous-wave Doppler ultrasound may be much more useful than conventional ultrasound and SF measurements in detecting fetuses at risk of stillbirth. The prevalence of AEDF in other LMIC settings needs to be confirmed, and a randomised trial will need to be performed to confirm its effect on mortality

\section{Conclusions}

The prevalence of AEDF of the umbilical artery in a low-risk pregnant population was $\sim 10$ times higher in the Mamelodi population than previously recorded in high-income countries. Use of the Umbiflow findings led to a reduction in the perinatal mortality rate, the 
reduction being greatest in macerated stillbirths. Umbiflow screening is potentially an effective way of screening all women for abnormal Doppler flow velocities to reduce unexpected fetal death.

Declaration. This study was part of SN's PhD thesis.

Acknowledgements. We wish to acknowledge Susan Mogale and Peggy Mahlangu from Stanza Bopape and Johanna Skhosana and Cynthia Mdlalose from Dark City for doing Umbiflow tests, Phendile Ntuli for tracing results, Cathy Bezuidenhout for data management, Prof. Hein Odendaal for reviewing the article, and Busisiwe Vilikazi from the CSIR for providing technical support for the Umbiflow apparatus. We also thank the SAMRC and the CSIR for funding this study.

Author contributions. SN helped develop the protocol, managed all the high-risk Umbiflow women, and helped with data analysis and with writing the article. JM performed the data analysis and helped with the writing of the article. TH assessed the false-negative rate and reviewed and edited the article. RCP conceived the study, developed the protocol, drafted the initial article and helped with the data analysis.

Funding. The Tshwane 'Khulelwe' Project, an integrated ICT-enabled community-oriented antenatal care study with Doppler ultrasound (Umbiflow) assessment, was funded $50 / 50$ by the South African Medical Research Council and the CSIR as part of the Strategic Health Innovation Partnerships. The CSIR provided technical support for the Umbiflow apparatus. The funders were not involved in data analysis or in writing the article.

Conflicts of interest. None.

1. Gardosi J, Madurasinghe V, Williams M, Malik A, Francis A. Maternal and fetal risk factors for stillbirth: Population based study. BMJ 2013;346:f108. https://doi.org/10.1136/bmj.f108

2. Katz J, Lee AC, Kozuki N, Lawn JE, et al. Mortality risk in preterm and small-for-gestational-age infants in low-income and middle-income countries: A pooled country analysis. Lancet 2013;382(9890):417 425. https://doi.org/10.1016/S0140-6736(13)60993-9
3. Chiswick ML. Intrauterine growth-retardation. BMJ 1985:291(6499):845-848.

4. Pilliod RA, Cheng YW, Snowden JM, Doss AE, Caughey AB. The risk of intrauterine fetal death in the small-for-gestational-age fetus. Am J Obstet Gynecol 2012;207(4):318.el-6. https://doi. org/10.1016/j.ajog.2012.06.039

5. Gardosi J, Mul T, Mongelli M, Fagan D. Analysis of birthweight and gestational age in antepartum stillbirths. Br J Obstet Gynaecol 1998;105(5):524-530. https://doi.org/10.1111/j.1471-0528.1998. tb10153.x

6. Allanson ER, Muller M, Pattinson RC. Causes of perinatal mortality and associated maternal complications in a South African province: Challenges in predicting poor outcomes. BMC Pregnancy Childbirth 2015:15:37, https//doiorg/10.1186/s12884-015-0472-9

7. Alfirevic Z, Stampalija T, Gytes GML. Fetal and umbilical Doppler ultrasound in high-risk pregnancies. Cochrane Database Syst Rev 2017, Issue 6. Art. No.: CD007529. https://doi.
pritick pregnancies. Cochrane Database Syst
org/10.1002/14651858.CD007529.pub4

8. Alfirevic Z, Stampalija T, Medley N. Fetal and umbilical Doppler ultrasound in normal pregnancy. Cochrane Database Syst Rev 2015, Issue 4. Art. No.: CD001450. https://doi.org/10.1002/14651858. CD001450.pub4

9. Villar J, Bảageei H, Piaggio G, et al., for the WHO Antenatal Care Trial Research Group. WHO antenatal care randomised trial for the evaluation of a new model of routine antenatal care. Lancet 2001;357(9268):1551-1564. https://doi.org/10.1016/S0140-6736(00)04722-X

10. World Health Organization, Department of Reproductive Health, Family and Community Health Integrated Management of Pregnancy and Childbirth. Geneva: WHO, 2003.

11. Theron GB, Theron AM, Odendaal HJ, Bunn AE. Comparison between a newly developed PC-based Doppler umbilical artery waveform analyser and a commercial unit. S Afr Med J 2005;95(1):62-64.

2. Pattinson RC, Theron GB, Thompson ML, Lai Tung M. Doppler ultrasonography of the fetoplacental circulation - normal reference values. S Afr Med J 1989;76:623-625.

13. Kiserud T, Piaggio G, Carroli G, et al. The World Health Organization Fetal Growth Charts: A multinational longitudinal study of ultrasound biometric measurements and estimated fetal weight. PLoS Med 2017:14(1):e1002220. https://doi.org/10.1371/journal.pmed.1002220

14. Odendaal HJ, Theron AM, Carstens MH, de Jager M, Grovè D. Intrauterine deaths in high-risk pregnancies with normal and borderline umbilical artery Doppler flow velocity waveforms. S Afr J Obstet Gynaecol 2008;14(1):33-37.

15. Everett K, Odendaal H, Steyn K. Doctors' attitudes and practices regarding smoking cessation during pregnancy. S Afr Med J 2005;95(5):350-354.

16. Lavin T, Preen DB, Pattinson R. Timing and cause of perinatal mortality for small-for-gestational age babies in South Africa: Critical periods and challenges with detection. Matern Health Neonatol Perinatol 2016;2:11. https://doi.org/10.1186/s40748-016-0039-4

17. Pattinson RC, Brink AL, de Wet PE, Odendaal HJ. Early detection of poor fetal prognosis by serial Poppler velocimetry in high-risk pregnancies. S Afr Med J 1991;80:428-431.

18. Karsdorp VH, van Vugt JM. Clinical significance of absent or reversed end diastolic velocity in the umbilical artery. Lancet 1994;344(8938):1664-1689. https://doi.org/10.1016/S01406736(94)90457-X

19. World Health Organization. WHO Recommendations on Antenatal Care for a Positive Pregnancy Experience. Geneva: WHO, 2016.

20. Goldenberg RL, Nathan RO, Swanson D, et al. Routine antenatal ultrasound in low- and middleincome countries: First look - a cluster randomised trial. BJOG 2018;125(12):1591-1599. https:// doi.org/10.1111/1471-0528.15287

Accepted 5 October 2018. 